Website: http://revistas.lamolina.edu.pe/index.php/acu/index

(C) Universidad Nacional Agraria La Molina, Lima - Perú

\title{
Alargamiento del raquis del racimo y modificación de algunas características de los frutos con aplicaciones de ácido giberélico en vid 'Red Globe' (Vitis vinifera)
}

\author{
Rachis elongation and modification of some berry characteristics as a result of gibberellic acid \\ treatments in 'Red Globe' grapevine (Vitis vinifera)
}

\author{
María P. Santolalla A. ${ }^{1}$; Jorge Escobedo A. ${ }^{2^{*}}$ \\ * Autor de correspondencia
}

\section{Resumen}

En Casma, Perú, racimos florales de vid 'Red Globe' en tres estados de desarrollo: E1, E2 y E3, correspondientes a los códigos 55, 57 y 62 de la escala $\mathrm{BBCH}$, fueron sumergidos en cuatro soluciones de $\mathrm{AG}_{3}: 10,20,30$ y 40 ppm, además de un testigo en agua sola, para evaluar en cosecha, su efecto en la longitud del raquis, el número y peso de frutos y el número de semillas. Con 20 y 40 ppm el raquis aumentó en $22,11 \mathrm{~cm}$ en promedio, con 10 y 30 ppm la media fue de $20,04 \mathrm{~cm}$, los testigos registraron 16,6 cm; aplicaciones a panículas más desarrolladas fueron mejores $(18,59,19,72$ y $22,06 \mathrm{~cm}$, para E1, E2 y E3). El número de frutos por racimo solo fue más elevado con 20 y 40 ppm (59 en promedio) en relación con los testigos (46); aplicaciones tardías tuvieron mejor efecto: 41, 53 y 62 bayas para E1, E2 y E3. E1 peso individual de los frutos disminuyó con todas las concentraciones de $\mathrm{AG}_{3}$ (7,56 g en promedio) en comparación con los testigos (9,91 g); aplicaciones en E2 produjeron menor peso de bayas $(6,66 \mathrm{~g})$ que en E1 y E3 (8,71 en promedio). El número de semillas por fruto fue mayor en los testigos $(2,72)$ disminuyendo con todas las concentraciones de $\mathrm{AG}_{3}: 1,95$, $1,55,1,17$ y 0,8 para 10, 20, 30 y 40 ppm; en E2, el efecto inhibitorio (1,21 semillas) fue más fuerte que en E1 y E3 (1,85 semillas en promedio).

Palabras clave: racimo; raquis; panícula; antesis; $\mathrm{AG}_{3}$; baya.

\begin{abstract}
In Casma, Perú, floral clusters of 'Red Globe' grapevine were dipped in four GA solutions: 10, 20, 30 and 40 ppm, besides a control in water alone, at three different stages of panicle development: E1, E2 and E3, corresponding to codes 55,57 y 62 of BBCH scale, to evaluate their effects on bunch rachis length, number and weight of fruits and number of seeds per fruit. Evaluations were made at the harvest. With 20 and 40 ppm GA increase in length of rachis was 22,11 $\mathrm{cm}$ on average, followed by 10 and $30 \mathrm{ppm}(20,04 \mathrm{~cm}$ on average), the control treatments reached 16,6 cm; applications to more developed panicles were better $(18,59,19,72$ and 22,06 cm, for E1, E2 and E3 stages). Number of fruits per bunch only was higher with 20 and 40 ppm (59 on average) compared to controls (46); development stages of panicles had a marked effect (41, 53 and 62 berries for E1, E2 and E3). Individual fruit weight was negatively affected by all GA concentrations (7,56 g on average), compared to controls $(9,91 \mathrm{~g})$; applications at E2 stage produced berries with lower average weight $(6,66 \mathrm{~g})$ than E1 and E3 (8,71 g on average). Berry seed number was higher in controls $(2,72)$ decreasing with all $\mathrm{GA}_{3}$ concentrations, registering $1,95,1,55,1,17$ and 0,8 seeds per fruit for 10,20, 30 and 40 ppm; treatments at stage E2 (1,21 seeds) had a stronger inhibitory effect compared to E1 and E3 stages (1,85 seeds in average).
\end{abstract}

Keywords: panicle; cluster; bunch; rachis; anthesis; GA.

\section{Introducción}

Las hormonas vegetales se producen en diversos tejido de las plantas e intervienen de manera equilibrada como un factor indispensable para que todos los procesos que corresponden a las etapas fenológicas ocurran de forma armónica (Castro y Vieira, 2001; Davies, 2004). Los niveles endógenos de determinada hormona básicamente dependen de la magnitud de su producción por la planta, y sus efectos, en gran medida, están condicionados a factores climáticos como luz y temperatura y también a la presencia a concentración de otras hormonas (Coll et al., 2001; Ruiz, 1.998). El empleo de reguladores de crecimiento (PGR) sintéticos, con efectos similares a las hormonas, es una práctica frecuente en fruticultura pues permite regular o modificar algunos procesos y generar cambios de algunas características físicas, químicas o metabólicas (Castro y Vieira, 2001; Taiz y Zeiger, 2004).

El grupo de reguladores más empleado en el cultivo de la vid es el de las giberelinas a través de aplicaciones exógenas del ácido giberélico, generalmente $\mathrm{AG}_{3}$. El nivel de la giberelina endógena al momento de realizar las aplicaciones de $\mathrm{AG}_{3}$ tiene un rol decisivo en los efectos que se esperan. En cultivares sin semilla la giberelina puede ser detectada en los frutos solo en etapas muy tempranas, 
durante los primeros 14 días después de la antesis. Por ello es que el nivel endógeno de la hormona en las bayas de estos cultivares puede ser un factor limitante de su desarrollo y responden más ampliamente a las aplicaciones en estados más avanzados de la primera etapa de su crecimiento (Coombe, 1960; Weaver, 1961; Lavee, 1987). En la industria de la uva de mesa las aplicaciones de $\mathrm{AG}_{3}$, con intensidades variables según los cultivares, forman parte obligatoria de su manejo comercial para alcanzar los requerimientos de calidad exigidos por el mercado, y generalmente se buscan tres objetivos: alargamiento de la inflorescencia, raleo de flores e incremento de tamaño de las bayas (Dokoozlian, 2000; Pires y Botelho, 2001; AbuZahra, 2010; Dimovska et al., 2011; Abu-Zahra y Salameh, 2012; Özer et al., 2012; Dimovska et al., 2014; Vyvervan der, 2016). El estado fenológico al momento de la aplicación es fundamental para obtener los resultados que se esperan y las épocas específicas, generalmente varían según los cultivares en función de la floración y el tamaño de las bayas (Christensen, 2000; Dokoozlian, 2000; Hed et al., 2011; Molitor et al., 2012).

En el cultivar con semilla Cabernet Sauvignon se ha detectado que entre dos y cuatro semanas después de plena floración, existen dos picos de producción de giberelinas, ambos en la fase I de desarrollo de las bayas y muy relacionados con el número de semillas (Scienza et al., 1978). Esto determina que los cultivares con semilla sean mucho más sensibles a las aplicaciones de $\mathrm{AG}_{3}$ en pre floración cuando la giberelina endógena aún no existe o es escaza, y por el contario, generalmente el efecto es pequeño o menos marcado en los tratamientos post floración (Lavee, 1987), momento en que hay giberelinas en abundancia producida por las semillas. Por eso es que de manera general, el empleo de giberelinas en cultivares con semilla es limitado (Dokoozlian, 2000). No obstante, existen una serie de reportes que informan de resultados variados encontrados en cultivares con semilla con aplicaciones de $\mathrm{AG}_{3}$ tempranas (crecimiento de racimos) e incluso tardías (después del cuajado). En 'Red Globe' por ejemplo se logra uniformidad y mayor peso de bayas cuando se emplean concentraciones de 20 y 40 ppm, dos semanas después del cuajado, en el momento en que los frutos tienen de 10 a 12 milímetros de diámetro (Roses y Valenzuela, 1999; Dokoozlian, 2000). En la variedad Italia, aplicaciones de entre 0,5 a $50 \mathrm{ppm}$ al inicio de floración dieron como resultado racimos más sueltos y alargados $\mathrm{y}$ bayas con menor número de semillas, sin embargo cuando las aplicaciones fueron más tardías (frutos de 8 $\mathrm{mm}$ de diámetro), se incrementó el peso de los racimos y las bayas, pero no hubo aumento de los sólidos solubles (Guerra et al., 1981; Leão, 2000; Feitosa, 2002). Otros resultados con esta misma variedad y con sus mutaciones Benitaka y Brasil, indican que el $\mathrm{AG}_{3}$ en concentraciones de 10 a 30 ppm aplicado directamente sobre los racimos entre 20 y 30 días después de la floración solo resultó en una mayor rigidez de la epidermis de los frutos y sus pedicelos, sin afectar significativamente el tamaño y forma de las bayas (Pires, 1998; Pires y Botelho, 2001). En otro cultivar con semillas como Niágara Rosada (Vitis labrusca), tampoco se modificaron las características del fruto ni de los racimos cuando se aplicó 100 ppm de $\mathrm{AG}_{3}$, 14 días después de la floración (Botelho et al., 2003). Resultados similares se reportan con el cv. Delaware, con 50 ppm aplicado antes y después de floración, pero cuando se agregó nitrato de amonio se incrementó el tamaño de los frutos por un aumento del volumen de las células y el contenido de sólidos solubles disminuyó ligeramente (Ungans et al., 2003).

Las variadas respuestas a la aplicación de giberelinas exógenas confirman que su efecto está supeditado tanto a factores internos como externos y por lo cual, los momentos y dosis para lograr los efectos deseados deben de ser precisados en función del cultivar y de las condiciones medioambientales, especialmente clima.

Red Globe es una de las principales variedades de uva de mesa que se cultiva en Perú y la que más se exporta. Sus bayas son rojas y redondas, de gran calibre, con pulpa carnosa y consistente y con tres a cuatro semillas. La planta es de poco a mediano vigor y de producción uniforme. Su fertilidad generalmente la presenta entre la quinta y sexta yema. El racimo se caracteriza por ser grande y suelto, con un pedúnculo largo y fino, y un pedicelo firme que otorga gran resistencia al desgrane (Muñoz y Lobato, 2000; Vaysse et al., 2001)

Bajo las premisas anteriores, se planificó el siguiente trabajo de investigación, teniendo como objetivo principal determinar, en las condiciones de la localidad de Casma, el efecto de cinco concentraciones y tres épocas de aplicación de ácido giberélico $\left(\mathrm{AG}_{3}\right)$ en el alargamiento del raquis y sobre otros parámetros relacionados con características de calidad del fruto del cultivar de uva de mesa 'Red Globe'.

\section{Materiales y métodos}

El experimento fue conducido en la localidad de Casma, Provincia de Casma, Departamento de Ancash, ubicado en la zona de la costa central del Perú a una altitud de 38 m.s.n.m., entre abril y septiembre del año 2010, periodo durante el cual se registraron temperaturas promedio de $18,9^{\circ} \mathrm{C}$. Las plantas experimentales del cultivar Red Globe injertadas sobre el portainjerto MGT 101-14, de tres años de edad, formaban parte de un lote comercial de 5 ha, conducido en el sistema de parrón español con distanciamientos de 3 x 2,5 m y regado por goteo. Después de la poda (12 abril) se seleccionaron y marcaron 75 plantas para el experimento.

Los dos factores en estudio y sus niveles correspondientes fueron los siguientes:

1. Concentración de $\mathrm{AG}_{3}$. Cinco niveles: $\mathrm{C} 0, \mathrm{C} 1, \mathrm{C} 2, \mathrm{C} 3$ y C4 que corresponden a 0 (testigo), 10, 20, 30 y 40 ppm.

2. Epoca de aplicación. Tres niveles: E1 (15 de mayo, inicio de crecimiento de panículas), E2 (29 de mayo, panículas totalmente formadas) y E3 (12 de junio, $20 \%$ de aperturas florales aproximadamente), equivalentes a los estados 55, 57 y 62 de la escala BBCH (Lorenz et al., 1995). 
Como resultado de lo anterior se conformaron 15 tratamientos:

$\begin{array}{lll}\text { 1. E1C0 (testigo) } & \text { 6. E2C0 (testigo) } & 11 . \mathrm{E} 3 \mathrm{C} 0 \text { (testigo) } \\ \text { 2. E1C1 } & \text { 7. E2C1 } & 12 . \mathrm{E} 3 \mathrm{C} 1 \\ \text { 3. E1C2 } & \text { 8. E2C2 } & 13 . \mathrm{E} 3 \mathrm{C} 2 \\ \text { 4. E1C3 } & 9 . \mathrm{E} 2 \mathrm{C} 3 & 14 . \mathrm{E} 3 \mathrm{C} 3 \\ \text { 5. E1C4 } & 10 . \mathrm{E} 2 \mathrm{C} 4 & 15 . \mathrm{E} 3 \mathrm{C} 4\end{array}$

Se emplearon cinco plantas por tratamiento y al inicio de brotamiento, en cada una se eligieron dos brotes y en cada uno de estos se marcó una inflorescencia como unidad experimental, de manera que cada tratamiento se evaluó en 10 inflorescencias. La aplicación del regulador del crecimiento se hizo entre 7 y 9 a.m., por inmersión individual de las panículas en las respectivas soluciones (método conocido como "jarreo") por aproximadamente cinco segundos, los testigos se sumergieron en agua sola

Las evaluaciones se realizaron a la cosecha y los parámetros que se evaluaron fueron; longitud del raquis, número de bayas por racimo, peso de las bayas y número de semillas por fruto. La longitud del raquis se expresó como incremento de longitud a partir de la medida inicial registrada el 14 de mayo, un día antes de la primera aplicación de la giberelina y la medida final, el día anterior a la cosecha. Para evaluar el peso de las bayas y el número de semillas, a la cosecha se tomaron ocho frutos por racimo (dos del tercio medio, cuatro de la parte central y dos del tercio inferior).

El arreglo estadístico fue un Diseño Completamente al Azar con un Análisis Combinado de dos factores $5 \mathrm{x}$ 3. Los datos fueron sometidos al análisis de varianza por el programa SAS, aplicando la prueba de Duncan para la comparación de medias.

\section{Resultados y discusión}

\section{$\underline{\text { Longitud del raquis }}$}

Considerando el incremento del tamaño del raquis del racimo como índice de su crecimiento, en todas las épocas de aplicación, los efectos combinados con las concentraciones de $\mathrm{AG}_{3}$ no provocaron alteraciones significativas en el tamaño del raquis. Sin embargo, como puede verse en la Figura 1, los promedios de las épocas muestran diferencias significativas entre ellas, con mayores incrementos a medida que las panículas estuvieron en estados más avanzados de desarrollo al recibir su respectiva aplicación, siendo la mejor, con 22,06 cm, cuando las panículas mostraban el inicio de las aperturas florales (E3). De la misma manera. independientemente de las épocas, el promedio de las concentraciones del regulador del crecimiento revela una influencia significativa de todas ellas en relación con los testigos, destacando $\mathrm{C} 2$ y C4 con incrementos de 22,31 y $21,92 \mathrm{~cm}$, respectivamente. Es posible que la aplicación más tardía coincida con el descenso de la giberelina endógena que llega al raquis debido a la madurez de las hojas cercanas y la lejanía cada vez mayor del ápice del brote en crecimiento en relación con las inflorescencias. En tales circunstancias el aporte de las aplicaciones exógenas puede ser significativo, pues tal como anota Scienza et al.
(1978), para ‘Cabernet Sauvignon', recién 2 y 4 semanas después de la plena floración, y muy relacionado con la presencia de las semillas, se presentan dos picos de producción de la hormona en los frutos recién formados.

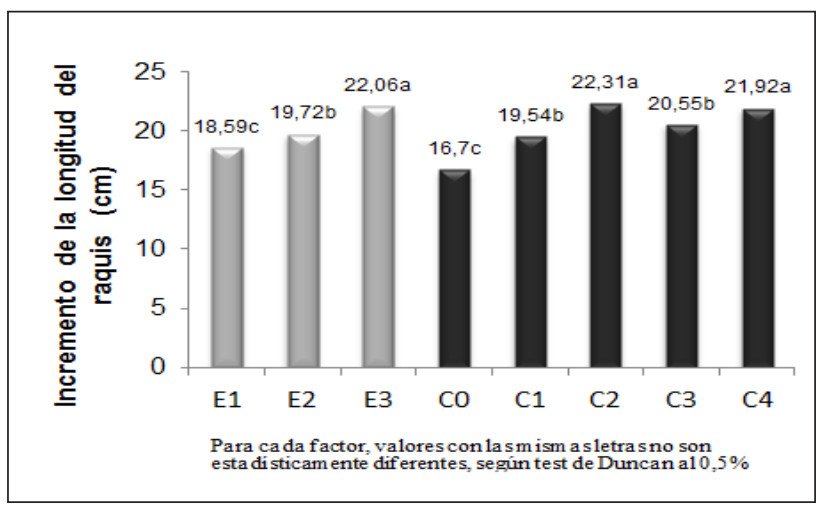

Figura 1. Incremento de la longitud del raquis del escobajo. Promedios de épocas de aplicación y concentraciones independientes
E1: Inicio de crecimiento de panículas E2: Panículas totalmente formada E3: Inicio de aperturas florales
C0: Testigo
C2: $20 \mathrm{ppm}$
C4: $40 \mathrm{ppm}$
C1: $10 \mathrm{ppm}$ C3: $30 \mathrm{ppm}$

El efecto positivo del $\mathrm{AG}_{3}$ en el alargamiento de los racimos está documentado desde hace varios años, tanto para variedades de mesa como para vino, pero la respuesta varía según las dosis, los cultivares y el momento de aplicación, aunque el mayor crecimiento del raquis y de los pedicelos parece que, como en el presente trabajo, se logra con aplicaciones tardías (Weaver y McCune, 1962; Benavente, 1988; Hernández y Vargas, 1993; Coelho et al., 2004, Koukourikou et al., 2015; Acheampong et al., 2017). No obstante, algunas veces, como lo reportado para los cultivares Tokay y Zinfadel por Weaver y Pool (1971), las aplicaciones de giberelinas para ralear frutos no tuvieron influencia en la longitud de los racimos.

\section{Número de bayas por racimo}

De acuerdo a lo que puede observarse en la Figura 2A, en la época de aplicación temprana (E1), las concentraciones de 10 y $30 \mathrm{ppm}$ dieron como resultado un menor número de bayas que alcanzaron diferencias estadísticas significativas en relación con el testigo, mientras que con las otras dos concentraciones las cifras fueron similares al testigo. Sin embargo en la segunda y tercera época de aplicación (E2 y E3), el efecto registrado fue un incremento del número de bayas de los racimos, que alcanzó diferencias estadísticas con los testigos para 30 ppm en E2 y para 20 y 40 ppm en E3.

Los promedios de cada factor, Figura 2B, indican, que un mayor número de bayas en los racimos fue influenciado por las dos últimas aplicaciones, destacando significativamente la más tardía que registró un promedio de 62 bayas por racimo. Igualmente más bayas se formaron por efecto de las concentraciones de 20 y 40 ppm (con 61 y 57 bayas). 


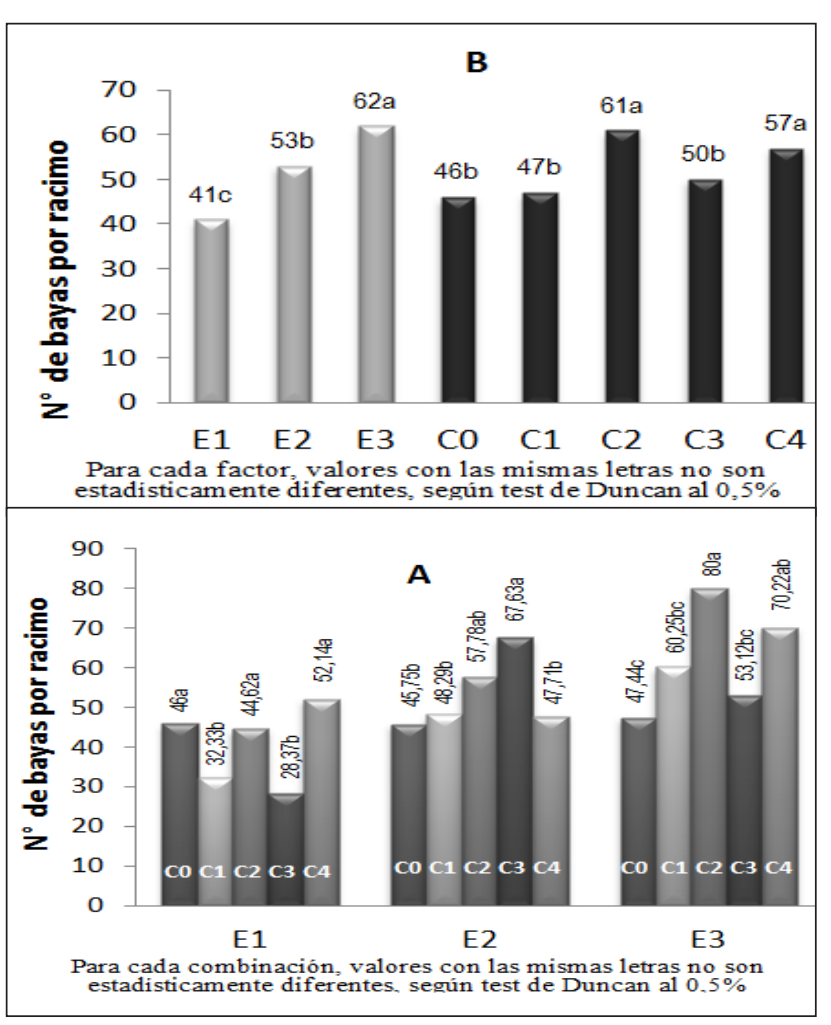

Figura 2. Número de bayas de racimos. A: Interacción de época de aplicación y concentraciones de $\mathrm{AG}_{3} \mathrm{~B}$ : Promedios de épocas y concentraciones independientes

$\begin{array}{lcc}\text { E1: Inicio de crecimiento de panículas } & \text { C0: Testigo } & \text { C1: } 10 \mathrm{ppm} \\ \text { E2: Panículas totalmente formadas } & \text { C2: } 20 \mathrm{ppm} & \text { C3:30 ppm } \\ \text { E3: Inicio de aperturas florales } & \text { C4: } 40 \mathrm{ppm} & \end{array}$

Existen reportes del efecto raleador de aplicaciones tempranas de $\mathrm{AG}_{3}$ en vides con semilla, como el de Leao (2000) quien trabajando específicamente con el cultivar Italia, encontró que con concentraciones de $\mathrm{AG}_{3}$ de hasta $50 \mathrm{ppm}$ aplicadas al inicio de floración se redujo el número de granos por racimo. En la presente invetigación el mayor raleo fue obtenido con la aplicación en una época mucho más temprana que la reportada por Leao (2000). Luego el efecto raleador fue perdiendo intensidad a medida que la edad los racimos al recibir los tratamientos fue más avanzada (coincidiendo con lo observado por Dass y Randhawa (1968) en diversos cultivares con semilla), para finalmente registrarse un aumento de las bayas, resultado este último que concuerda con lo encontrado por Pires (1998) que indica que con $\mathrm{AG}_{3}$ aplicado al cultivar Maria en plena floración (un poco más tarde que el estado E3) se obtuvo un incremento significativo en el número de frutos. En 'Gros Colman', otro cultivar con semillas, las aplicaciones 5-6 días antes de plena floración también incrementaron el número de frutos cuajados (Dass $\mathrm{y}$ Randhawa, 1968).

\section{Peso de las bayas}

No se encontró diferencias significativas para las interacciones de los dos factores en estudio. Sin embargo el promedio independiente de los mismos anotado en la Figura 3, muestra que en comparación con los testigos que alcanzaron 9,91 gramos, todas las concentraciones del regulador, sin diferencias entre ellas, afectaron negativamente el peso de las bayas (entre 8,15 y 6,76 g). El promedio de las épocas revela que las aplicaciones en la segunda época fueron más restrictivas.

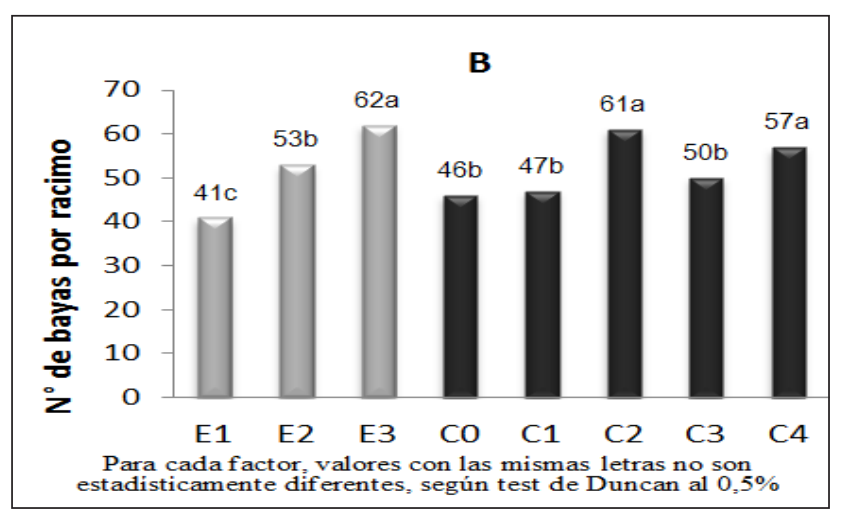

Figura 3. Peso de bayas de los racimos. Promedios independientes de épocas de aplicación y concentraciones de $\mathrm{AG}_{3}$

E1: Inicio de crecimiento de panículas E2: Panículas totalmente formadas E3: Inicio de aperturas florales

$$
\begin{array}{ll}
\text { C0: Testigo } & \text { C1: } 10 \mathrm{ppm} \\
\text { C2: } 20 \mathrm{ppm} & \text { C3: } 30 \mathrm{ppm} \\
\text { C4: } 40 \mathrm{ppm} &
\end{array}
$$

En un racimo, existe una relación inversa entre número de bayas y el peso individual de cada una de ellas. Esto se refleja de manera general en los resultados obtenidos, es decir el mayor peso de las bayas corresponden a racimos con menor número de frutos y viceversa. En los cultivares con semilla, otro factor involucrado en el peso de los frutos es el contenido de semillas. Por lo general las bayas más grandes poseen más semillas. Los resultados relacionados con este último parámetro, que se muestran más adelante, parecen confirmar esta relación.

Las referencias son variadas y parece que con aplicaciones de giberelina en estados fenológicos más avanzados de floración los resultados pueden favorecer el peso de los frutos en variedades con semillas, tal como informan Dokoozlian (2000) y Roses y Valenzuela (1999) quienes encontraron un mayor peso de las bayas en 'Red Globe' cuando se aplicaron 20 y 40 ppm en época mucho más tardía, dos semanas después del cuajado. Otros resultados con aplicaciones antes y después de floración en cultivares como Niágara Rosada, Delaware, Italia y dos de sus mutaciones, señalan que con concentraciones entre 10 y 100 ppm no se afectó ni el tamaño ni la forma de los frutos (Pires, 1998; Pires y Botelho, 2000; Botelho et al., 2003, Ungans et al. 2003).

\section{Número de semillas}

En la Figura 4A se encuentra registrado el número promedio de semillas en las bayas de los racimos que recibieron los tratamientos resultantes de las interacciones 
entre épocas de aplicación y concentraciones de $\mathrm{AG}_{3}$. En las tres épocas se puede observar que hay reducción de la cantidad de semillas por efecto de la giberelina empleada. En la primera época, alcanzan diferencias significativas las concentraciones de 20 y $40 \mathrm{ppm}$ de $\mathrm{AG}_{3}$ con las cuales se logra 1,6 y 1,1 semillas por fruto, respectivamente, en comparación con 3,1 del testigo. Con las aplicaciones de la segunda época la reducción es más acentuada, registrándose $0,8,0,0$ y 0,7 semillas por baya para las concentraciones de 20, 30 y 40 ppm, respectivamente en comparación con el testigo que tuvo 2,6 semillas. Con la aplicación más tardía (E3) solo la concentración más elevada de la giberelina, 40 ppm produjo frutos con menos semillas que el testigo $(0,5$ y 2,4 respectivamente).

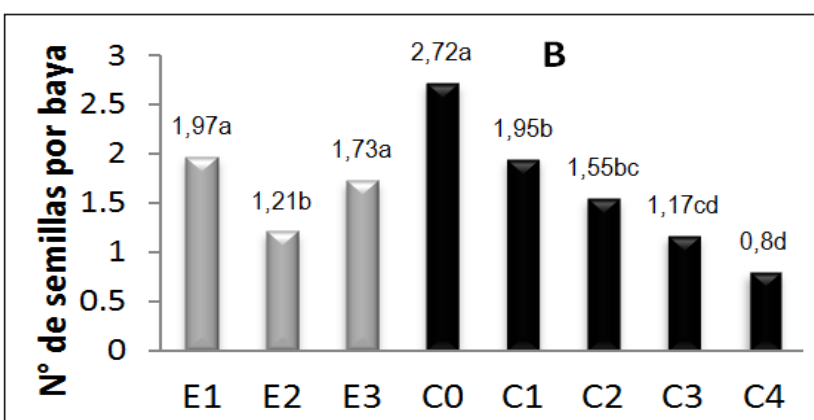

Para cada factor, valores con las mismas letras no son estadísticamente diferentes, según test de Duncan al 0,5\%

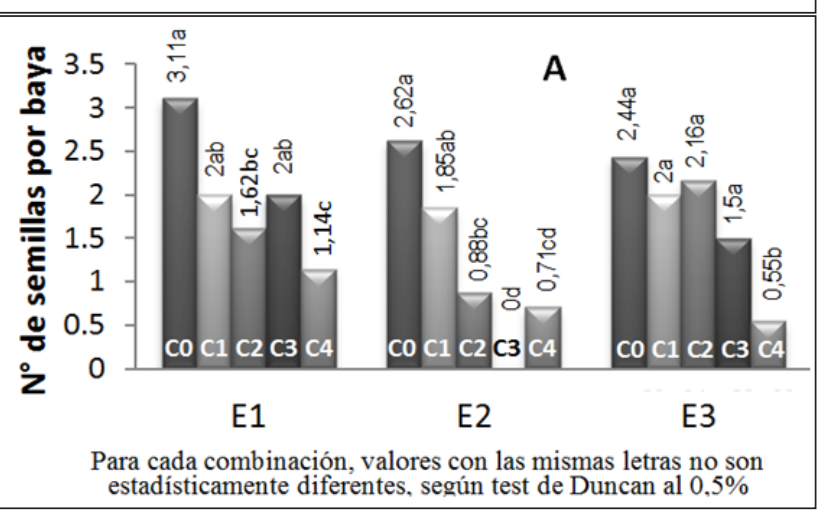

Figura 4. Número de semillas por baya. A: Interacciones entre épocas de aplicación y concentraciones de $\mathrm{AG}_{3}$. B: Promedios independientes de épocas de aplicación y concentraciones

E1: Inicio de crecimiento de panículas E2: Panículas totalmente formadas

E3: Inicio de aperturas florales

C0: Testigo $\mathrm{C} 2: 20 \mathrm{ppm}$ C4: 40 ppm

$\mathrm{C} 1: 10 \mathrm{ppm}$ C3: $30 \mathrm{ppm}$

El promedio de los factores independientemente uno del otro, Figura 4B, indica que con todas las concentraciones de $\mathrm{AG}_{3}$, y con diferencias estadísticas entre ellas, hubo en las bayas un menor número de semillas (entre 1,95 y 0,8) en relación con el testigo (2,72 semillas). Esta reducción fue mayor a medida que la dosis de giberelina aplicada fue más concentrada. El promedio de la época E2 $(1,21$ semillas) tuvo un mayor efecto reductor de semillas que la más temprana y la más tardía (1,97 y 1,73 semillas y sin diferencias significativas entre estas).
La inducción a la apirenia por aplicaciones de giberelina a inicios de floración o en plena floración es referida por muchos autores (Gil y Escobar, 1979; Pires, 1998; Pires y Botelho, 2002; Santos et al., 2003; Taiz y Zeiger, 2004). En aplicaciones pre-floración, que es el caso de la presente investigación, posiblemente, el regulador actúe directamente sobre las estructuras celulares de los óvulos provocando insuficiente desarrollo de los mismos y también una pobre germinación de los granos de polen fallando así la fertilización de los óvulos, tal como indican Sugiura e Inaba (1968). En el mismo sentido, Okamoto y Miura (2005) estudiando el marcado efecto del ácido giberélico en la producción de fruta sin semilla en el cv. Delaware (naturalmente con semillas) encontraron que aplicado tempranamente, 12-17 días antes de plena floración produjo una inhibición del crecimiento del tubo polínico en los pistilos que sería resultado de la producción de inhibidores por los tejidos del ovario que recibieron el tratamiento.

Con aplicaciones más tardías, los mecanismos parecen ser diferentes. Cheng et al. (2013) trabajando con dos cultivares con semilla, Red Globe y Kyoho, encontraron que con tratamientos de giberelina efectuados 15 días después de plena floración, la fertilización ocurrió normalmente pero después se produjo un completo aborto de semillas aparentemente por daños celulares producto un desbalance funcional de reducción-oxidación promovido por la giberelina aplicada.

Si bien es cierto la reducción del número de semillas a través de la aplicación de giberelina puede ser un aspecto positivo importante en la calidad de las uvas, el peso de las mismas (y lógicamente su tamaño), es afectado negativamente, tal como se puede apreciar en la Figura 3.

\section{Conclusiones}

La concentración de $\mathrm{AG}_{3}$ incrementa la longitud del raquis, siendo el 20 y 40 ppm los mejores. Las aplicaciones más tardías tienen mayor efecto. A medida que las aplicaciones de $\mathrm{AG}_{3}$ son tardias, el número de bayas por racimo se incrementa. Siendo las concentraciones de 20 y 40 ppm las que producen el mayor número de frutos por racimo. Todas las concentraciones de $\mathrm{AG}_{3}$ afectan negativamente el peso de las bayas, siendo el efecto más notorio a medida que la cantidad del regulador aplicada fue mayor. La aplicación en la segunda época (panículas totalmente formadas) fue la que más afectó el peso de los frutos.

El número de semillas se reduce con las aplicaciones en todas las épocas, manifestándo un efecto más fuerte con los tratamientos de la segunda época. Las concentraciones más elevadas produjeron en promedio una mayor reducción de las semillas de los frutos.

\section{Literatura citada}

Abu-Zahra, T. 2010. Berry size of Thompson Seedless as influenced by the application of gibberellic acid and cane girdling. Pak. J. Bot., 42(3): 1755-1760, 2010. 
Abu-Zahra, T. and Salameh, N. 2012. Influence of Gibberellic acid and cane girdling on berry size of Black Magic grape cultivar. Middle-East J. Sci. Res. 11(6): 718-722.

Acheampong, A.; Zheng, C.; Halaly, T.;Giacomelli, L.; Takebayashi, Y.; Jikumaru, Y.; Kamiya, Y.; Lichter, A. and Or, E. 2017. Abnormal endogenous repression of GA signaling in a seedless table grape cultivar with high berry growth response to GA application. Front. Plant Sci., 24 May 2017.

Benavente, E. 1988. El uso del ácido giberélico en uvas de mesa. Aconex 21: 11-13.

Botelho, R.; Pires, E.; Terra, M. e Carvalho, C. 2003. Efeitos do thidiazuron e do ácido giberélico nas características dos cachos e bagas de uvas 'Niagara Rosada' na região de Jundiaí-SP. Revista Brasileira de Fruticultura, 25 (1): 96-99.

Christensen, L. 2000. Growth Regulators. Raisin Production Manual. pp. 139-141.

Castro, P. e Vieira, E. 2001. Aplicações de Reguladores Vegetais na Agricultura Tropical. Guaíba: Agropecuária. $132 p$.

Cheng, C.; Xu, X.; Singer, S.; Li, J.; Zhang, H.; Gao, M.; Wang, L.; Song, J. and Wang, X. 2013. Effect of GA treatment on seed development and seed-related gene expression in grape. PLoS One. 8(11).

Coelho, P.; José, D. e Gomez, E. 2004. Efeitos do anelamento e da aplicação de ácido giberélico e Crop Set sobre as características dos cachos de uvas sem sementes no vale do São Francisco. Seminário Novas Perspectivas para o cultivo da Uva sem Sementes, Embrapa Semi-Árido, Petrolina- Pernambuco, Brasil. Documentos 185p.

Coll, J.; Rodrigo, G.; Garcia, B.; y Tamés, R. 2001. En: Fisiologia Vegetal. Madrid. Ediciones Pirámide. 566p.

Coombe, B. 1960. Relationship of growth and development to changes in sugars, auxins, and gibberellins in fruit of seeded and seedless varieties of Vitis vinifera L. Plant Physiology, 35: 241 - 250.

Dass, H. \& Randhawa, G. 1968. Response of certain seeded Vitis vinifera varieties to gibberellin application at post bloom stage. Amer. J. Enol. Vitic., 19: 56-62.

Davies, P. 2004. Plant Hormones: Biosynthesis Signal Transduction, Action. 2004. $3^{\text {rd }}$ Ed.; Kluwer Academic Publishers, Dordrecht. 705p.

Dimovska, V.; Ivanova, V.; Ilieva, F. and Sofijanova, E. 2011. Influence of bio regulator gibberellic acid on some technological characteristics of cluster and berry from some seedless grape varieties. Journal of Agricultural Science and Technology B1:054-1058.

Dimovska, V.; Ivanova, V.; Salamovska, A. and Ilieva, F. 2014. Flame seedless grape variety (Vitis vinifera 1.) and different concentration of gibberellic acid $\left(\mathrm{GA}_{3}\right)$. Bulgarian Journal of Agricultural Science, 20(1):137142.
Dokoozlian, N. 2000. Plant growth regulators use for table grape production in California. 4th Int. Table Grape Symp. 129-143p.

Feitosa, C. 2002. Efeitos do CPPU y $\mathrm{AG}_{3}$ no cultivo de uva 'Italia' na região do submédio São Francisco, Nordeste do Brasil. Revista Brasileira de Fruticultura, 24(2): 348353.

Gil, G. y Escobar, R. 1979. Empleo del ácido giberélico para regular la compactación de racimos en parronales vigorosos. Ciencia e Investigación Agraria, 6(1): 15-20.

Guerra, M.; Barcellos, F.; Koller, O. 1981. Influência do ácido giberélico, aplicado em floração e pós-floração, sobre as características do cachos da videira Itália (Vitis vinifera L.). In: Congreso Brasileiro de Fruticultura, 4: 1278-1286.

Hed, B.; Ngugi, H. and Travis, J. 2011. Use of gibberellic acid for management of bunch rot on Chardonnay and Vignoles grape. Plant Dis. 95 (March): 269-278.

Hernández, A. y Vargas, L. 1993. Efecto del ácido giberelico sobre la compactación del racimo de las vides "Mourvedre" y "Syrah". Revista de la Facultad de Agronomía, Universidad de Zulia, Maracaibo, Venezuela, 10(2).

Koukourikou, M.; Zioziou, E.; Pantazaki, A.; Nikolaou, N. and Kyriakidis, D. 2015. Effects of gibberellic acid and putrescine on 'Thompson Seedless' grapes. American International Journal of Biology, 3(2): 19-29.

Lavee, S. 1987. Use fullness of growth regulators for controlling vine growth and improving grape quality in intensive vineyards. Acta Horticulturae, Davis, 206: 89-108.

Leão, P. 2000. Manejo e tratos culturais da videira. Petrolina, PE: Embrapa Semi-Árido, 2000. Não paginado. Apostila apresentada no Curso sobre Manejo da Cultura e Agronegócio da Uva de Mesa, Petrolina, PE.

Lorenz, D.H.; Eichhorn, K.W.; Bleiholder, H.; Klose, R.; Maier, U. and Weber, E. 1995. Phenological growth stages of the grapevine (Vitis vinifera L. ssp. vinifera)Codes and descriptions according to the extended BBCH scale. Austr. J. of Grape and Wine Research, 1, 100-103.

Molitor, D.; Behr, M.; Hoffinann, L. and Evers, D. 2012. Research note: Benefits and drawbacks of prebloom applications of gibberellic acid $\left(\mathrm{GA}_{3}\right)$ for stem elongation in Sauvignon Blanc. South African J. Enol. Vitic. 33(2): 198-202.

Muñoz I. y Lobato A. 2000. Principales cultivares. En: Valenzuela J. ed. Uva de mesa en Chile. Gobierno de Chile. Ministerio de Agricultura. Instituto de Investigaciones Agropecuaria. Chile. P.43-59p.

Okamoto, G. and Miura, K. 2005. Effect of pre-bloom GA application on pollen tube growth in cv. Delaware grape pistils. Vitis, 44 (4): 157-159.

Özer, C.; Semih, A.; Ergonul, O. and Aydin, S. 2012. 
The effects of berry thinning and gibberellin on Recel Uzumu table grapes. Pakistan Journal of Agricultural Sciences, 49(2): 105-112.

Pires, E. 1998. Emprego de reguladores de crescimento em viticutura tropical. Informe Agropecuário, 19(194): 4043.

Pires, E. y Botelho, R. 2001. Uso de reguladores vegetais na cultura da videira. In: Simpósio Brasileiro de uvas de mesa, 2000. Ilha Solteira. Anais (s. n.), 2001. 129-147p.

Pires, E. y Botelho, R. 2002 Emprego de reguladores de crescimento em viticultura. In: Viticultura e Enologia: atualizando conceitos. Belo Horizonte: Epamig - Fecd. 59-81p.

Roses, H. y Valenzuela, J. 1999. Uva de mesa en Chile, Red Globe y Crimson Seedless. Tierra adentro, 27: 22 -25 .

Ruiz, V. 1998. Fitorreguladores. En: Los Parásitos de la Vid: Estrategias de Protección Razonada, 4 ${ }^{\text {ta }}$ ed.: Mundi-Prensa, Madrid. 303-306p.

Santos, R.; Santos, H.; Venturin, M. e Camargo, U. 2003. Influência de doses e épocas de aplicação de ácido giberélico sobre o desenvolvimento do traço de semente em uva apirênica. Em: Congresso Brasileiro de Fisiologia Vegetal. Resumos: Brazilian Journal of Plant Physiology, Atibaia/SP, 15: 191.

Scienza, A.; Miravalle, C.; Visal, M. and Fregoni. 1978. Relationship between seed number, gibberellin and abscisic acid levels and ripening in Cabernet Sauvignon grape berries. Vitis. 17: 361 .

Sugiura, A. \& Inaba, A. 1968: Studies on the mechanism of gibberellin induced seedlessness of Delaware grapes. II. Development of ovules and pollen as affected by prebloom gibberellin treatment. Mem. Coll. Agric. Kyoto Univ., 93: 43-5.

Taiz, L. y Zeiger, E. 2004. Fisiologia Vegetal. $3^{\text {ra }}$ ed. Porto Alegre. $719 \mathrm{p}$

Ungans, M.; Kato, K.; Takemura, K.; Hori, T.; Ohara, H.; Ohkawa, K.; Matsui, H. and Bukovac, M. 2003. Effects of the combination of gibberellic acid and ammonium nitrate on the growth and quality of seedless berries in 'Delaware' grape. Journal of the Japanese Society for Horticultural Science, 72(5): 366-371.

Vaysse P., Charmont S., Audubert A., Marion M., Thiault J. F., Scandella D. and Bergounoux F. 2001. Recognizing table grape varieties. Reconnaître les varieties de raisin de table. Éditions Centre technique interprofessionnel des fruits et legumes. Paris, Francia. 69 p.

Vyver-Van der, L. 2016. The influence of gibberellic acid (GA $\square$ ) for berry thinning and berry sizing on table grape production, quality and fertility of Prime. Thesis presented in partial fulfilment of the requirements for the degree of Master of Agricultural Science at Stellenbosch University. December 2016.

Weaver, R. 1961. Growth of grapes in relation to gibberellin. In: Goulc, R. F. (ed.). Gibberellins: advances in chemistry series, n. 28. American Chemical Society: Washington. 89-108p.

Weaver, R. and McCune, S. 1962. Studies on prebloom sprays of gibberellin to elongate and loosen clusters of 'Thompson Seedless' grapes. American Journal of Enology and Viticulture, Davis, 13: 15-19.

Weaver, R.; Pool, R. 1971. Thinning 'Tokay' and 'Zinfandel' grapes by bloom sprays of gibberellin. Journal American Society for Horticultural Science. 96(6): 820-822. 